\title{
Context, interest relativity and the sorites
}

\author{
JASON STANLEY
}

According to what I will call a contextualist solution to the sorites paradox, vague terms are context-sensitive, and one can give a convincing dissolution of the sorites paradox in terms of this context-dependency. The reason, according to the contextualist, that precise boundaries for expressions like 'heap' or 'tall for a basketball player' are so difficult to detect is that when two entities are sufficiently similar (or saliently similar), we tend to shift the interpretation of the vague expression so that if one counts as falling in the extension of the property expressed by that expression, so does the other. As a consequence, when we look for the boundary of the extension of a vague expression in its penumbra, our very looking has the effect of changing the interpretation of the vague expression so that the boundary is not where we are looking. This accounts for the persuasive force of sorites arguments.

Suppose we are presented with fifty piles each of which has one grain less than the pile to its left. On the far left is a pile we are strongly inclined to call a heap when presented alone. This pile is in the definite extension of 'heap'. On the far right is a pile we are slightly inclined not to call a heap when presented alone. It is towards the end of the penumbra of 'heap'. But starting with the left-most pile (pile 1), we may make progressive judgements of the form:

(a) If that pile 1 is a heap, then pile 2 is a heap.

(b) If pile 2 is a heap, then pile 3 is a heap.

(c) Etc.

(d) Etc.

(n) If pile 49 is a heap, then pile 50 is a heap.

Each of these conditionals would be one to which we would assent. By agreeing to this succession of conditionals, we eventually come to pile number 50, which we now would strongly feel inclined to count as a heap. The explanation for this, according to the contextualist, is that as we are presented with each conditional, the two piles are sufficiently similar so as to cause us to interpret 'heap' in such a way to count one as a heap if and only if the other is a heap. This has the effect of changing 
the property expressed by 'heap' as we progress through the series of conditionals. $^{1}$

Before we evaluate the conditionals, the property expressed by 'heap' may exclude the last ten piles from counting in the extension of 'heap'. But once we start to evaluate the conditionals, the boundary for 'heap' changes. And that is why a boundary between the heaps and the non-heaps is undetectable. Wherever we look, it is not there.

In this paper, I will argue that the contextualist account of the sorites paradox is problematic. I will then turn to a close cousin of the contextualist account of the sorites, the interest-relative theory of vagueness advocated by Graff (2000). On Graff's view, vague terms are one and all context-sensitive, but not in a way that the contextualist believes. Graff's view evades the objections I give against the contextualist. In the second half of the paper, I argue that the mechanisms that allow Graff's interestrelative view to evade the problems facing the contextualist land her theory in perhaps more intractable difficulties.

Scott Soames, a prominent advocate of contextualism, states the contextualist position on the semantics of vague expressions as follows:

To say that vague predicates are context sensitive is to say that they are indexical. While the semantic content of an indexical varies from one context of utterance to another, its meaning does not. Rather its context-invariant meaning constrains the indexical to take on semantic contents with certain specified features. Sometimes these constraints identify semantic content in terms of a fixed contextual parameter - e.g. the content of ' $\mathrm{I}$ ' is the agent of the context, the content of 'now' is the time of the context, and the content of 'actually' is the world of the context. In other cases, the meaning of an indexical constrains its semantic content to be one that satisfies a certain condition - e.g. the content of 'he' must be male, the content of 'she' must be female, and the content of 'we' must be a group of individuals that includes the agent of the context. A speaker using one of these indexicals is free to select any salient content that satisfies the relevant constraints.

1 The contextualist solution faces something of a problem with conditionals the antecedent of which concerns the last member of the penumbra of a vague term, and the consequent of which concerns the first member of the determinate anti-extension. Some of her principles would lead her to count the consequent as true, while others would lead her to reject the consequent as false (essentially, those principles that are sensitive to the similarity between the two cases would rule in favour of the truth of the consequent, whereas the principles that require the members of the definite antiextension never to be in the extension of the term in any context would rule in favour of its falsity). This problem is raised in Robertson (2000). For Soames's reply, see Soames 2002: 443-44, n. 13. 
If, as I believe, vague predicates are context-sensitive, then this is the model on which they must be understood. (Soames 2002: 445) ${ }^{2}$

So, on the contextualist theory, each vague term is an indexical, like 'I', 'here', and 'now'.

Here is a fact about indexical expressions. Indexicals have invariant interpretations in Verb Phrase ellipsis (henceforth, VP ellipsis). So, for example consider:

(1) John likes me, and Bill does too.

(2) Hannah lives here, and Bill does too.

(3) Hannah is supposed to be in Syracuse now, and Mary is too.

(4) John saw Hannah's film, and Bill did too.

(5) John read that, and Bill did too.

There is no available interpretation of (1) in which John and Bill are said to like different people. This is so, even if the person who uttered the second conjunct of (1) is different than the person who uttered the first conjunct. Similarly, there is no available interpretation of (2) according to which Hannah and Bill are said to live in different places, and no interpretation of (3) according to which Hannah and Mary are supposed to be in Syracuse at different times. (4) cannot be uttered with the intention of expressing the proposition that John saw the film Hannah produced, while Bill saw the film Hannah directed. Finally, if one utters the first conjunct of (5) while demonstrating one object, say a book, the second conjunct must be understood expressing the proposition that Bill read that self-same book, even if one is pointing at a different book when uttering the second conjunct. ${ }^{4}$

When a pronominal expression has a non-invariant interpretation in VP ellipsis, this signals that a higher operator binds it. So, consider:

(6) John loves his mother, and Bill does too.

Suppose, in uttering (6), the speaker is pointing at Tim, and hence using 'his' demonstratively. In this case, (6) can only mean (7). But if 'his' is a pronoun anaphoric on 'John', then (6) can mean (8):

2 Other prominent contextualist theories include Kamp 1981 and Raffman 1994, 1996. It is not clear to what degree Tappenden's influential (1993) counts as a contextualist theory in the sense in which I have defined it here.

3 To be more exact, the contextualist aspect in Soames's (1999) theory is intended as a solution of the dynamic or particularized version of the sorites paradox, where speakers are confronted with a sorites series over a period of time. Other aspects of Soames's views play a more central role in addressing generalized versions of the sorites paradox. I will only be considering particularized versions of the sorites paradox in this paper.

${ }^{4}$ The one exception to this generalization about indexicals occurs with the discourse 'I love you. [response] I do too.' This does not work with verbs other than 'love'. 
(7) John loves Tim's mother, and Bill loves Tim's mother.

(8) John loves John's mother, and Bill loves Bill's mother.

The standard account of this ambiguity is that the logical form of the verb phrase in (6), when read as (8), is ' $\lambda x$ ( $x$ loves $x$ 's mother)'. This predicts reading (8) of (6), since (6), at logical form, is then:

(9) John $\lambda x$ (x loves $x$ 's mother), and Bill $\lambda x$ (x loves $x$ 's mother).

In sum, then, when an expression is used indexically or demonstratively, then it has an invariant reading under VP ellipsis. If, in contrast, it is used as an anaphor, then it is bound by a higher operator, and may give rise to non-invariant readings. ${ }^{5}$

According to the contextualist, a vague predicate has a character that determines different properties in different contexts. So, the content of a vague predicate must be invariant in VP ellipsis. But this raises problems in accounting for all versions of the sorites paradox. For consider the following version of the paradox. Suppose we are presented with a series of $n$ piles of grains. The $i+1$ th heap in the series of piles has one less grain than the $i$ th heap. The first member of the series clearly is a heap, and some grains that clearly do not suffice to make a heap form the nth member of the series. Suppose, ostending each pile of grains in turn (where 'that'; is a demonstrative used to refer to the $j$ th heap in the series), we say either:

(10) That ${ }_{1}$ is a heap, and that 2 is too, and that 3 is too, and that 4 is too, ... and that ${ }_{n}$ is too.

(11) If that ${ }_{1}$ is a heap, then that ${ }_{2}$ is too, and if that 2 is, then that 3 is, and if that ${ }_{3}$ is, then that $t_{4}$ is, ... and then that ${ }_{n}$ is.

The contextualist presumably wishes to claim that the reason each conjunct (10) is so compelling, and each conditional in (11), is that the similarity between the $i$ th member of the series and the $i+1$ th member of the series causes us to adjust the content of 'heap' so that its extension includes both. But since VP ellipsis is used in this version of the sorites, this strategy cannot succeed. If the word 'heap' is an indexical, then it does not shift its denotation in any of the different conjuncts in (10) or any of the different conditionals in (11). Since no context-shifts are possible in these cases, the contextualist's semantic account fails to explain why we find each step in (10) and (11) so compelling.

5 As Ludlow (1989) and Stanley (2000) emphasize, comparison classes for comparative adjectives are best thought of as the values of variables in logical form that can be anaphorically controlled by higher operators. So, in 'That elephant is small, and that flea is too', the logical form is something like 'That elephant $(\lambda x \lambda \mathrm{Y}(x$ small for $\mathrm{Y}))$, and that flea $(\lambda x \lambda \mathrm{Y}(x$ is small for $\mathrm{Y}))$.', where the ' $\mathrm{Y}$ ' argument is saturated by the common noun. Stanley (2000) provides evidence that higher quantifiers can bind the comparison class variable. 
The sorites paradox does not just arise for nouns such as 'heap'. For example, consider the verb 'shout'. One might suppose that if someone is shouting, then someone who is speaking a tenth of a decibel less loudly is also shouting. Suppose we start by considering someone who is clearly shouting, and in turn hear a series of people, each of whom is speaking a tenth of a decibel less loudly than the previous person. Then, we can say:

(12) If she ${ }_{1}$ is shouting, then she $e_{2}$ is too, and she 3 is too, and she 4 is too ...

Here, too the contextualist would have to maintain that the verb 'shout' changes its content in the successive occurrences of VP ellipsis. But, as we have seen, this is semantically implausible. Similar particularized versions of the sorites paradox can be constructed for prepositions, such as 'near' and 'far'. 6

So, the contextualist cannot explain these versions of the sorites paradox. But neither can she give a distinct explanation of (10)-(12). For presumably, we find each conjunct in (10) and (12) (and each conditional in (11)) compelling for the very same reason that we find each step in a normal sorites series compelling. There is no plausibility to a disjunctive explanation of these cases.

Any response the contextualist gives must be consistent with the fact that switching interpretations under VP ellipsis is not possible with other indexicals. For example, suppose Hannah, Mark, Sally and Bill are in my office. Pointing to each in turn, I utter:

(13) Hannah is here, and Mark is too, and Sally is too, and Bill is too.

In (13), the content of 'here' cannot shift under the VP ellipsis. For example, the space I indicate with 'here' cannot expand as I proceed down the sequence. So any appeal to special mechanisms that allow 'heap' to shift its interpretation in (10) and (11), and 'shout' in (13), must be consistent with the fact that 'here' cannot shift its interpretation in (12).

Nor is there plausibility in the claim that we reinterpret the original use of 'heap' or 'shout' with each successive VP ellipsis. First, in the case of (13), the successive VP ellipses do not allow the speaker to widen successively the original denotation for 'here'. Secondly, this response seems inconsistent with the phenomenology of the case. We certainly seem to be

${ }^{6}$ I emphasize that the sorites paradox can be constructed across syntactic categories, because the advocate of contextualism might appeal to features of nouns to respond to the above arguments. For example, the 'Nominal Restriction Theory' of quantifier domains advocated in Stanley and Szabo (2000) and Stanley (2002) might be brought in to address the potential context-dependency of 'heap'. This strategy is not available for verbs or prepositions. Furthermore, the strategy of appealing to domain restriction here is not compelling. 
using the ellipsis to make anaphoric reference to a property we have been attributing all along to other piles of grains. Finally, contextualism is intended as a semantic solution to the sorites. The solution is supposed to be that vague terms are semantically context-sensitive. The semantic content of each ellided phrase in the examples in (10)-(12) is determined by the semantic content of the initial use of the term, rather than any alleged reinterpretation of it. There is thus no contextualist semantic solution that treats all of these cases.

Intuitively, here is the problem versions of the sorites like (10)-(12) pose for the contextualist account. Owing to the use of VP ellipsis, the property expressed by the vague term in question must be the same across the different stages of the sorites series. But contextualist solutions account for the force of the sorites by appealing to a change in the property expressed. This explanation does not work for these examples.

The contextualist account also makes incorrect predictions about versions of the sorites paradox that involve counterfactual conditionals. Consider

(14) Patch P1 is red. That would still have been true even if P1 were indistinguishably more orange than it is. And that would still have been true even if $\mathrm{P} 1$ were indistinguishably more orange than that ... And that would still have been true even if P1 were indistinguishably more orange than that. But then it would have been clearly orange. Contradiction. ${ }^{7}$

According to the contextualist account, 'red' is an indexical expression. So its content is fixed in context. Subsequent propositional anaphora on the content of 'Patch P1 is red' is then fixed by the content of 'red' relative to the context of use of the first sentence in (14). So one of the counterfactual conditionals in (14) should seem to us to be false. But each counterfactual conditional in (14) is compelling, and presumably for the very same reason as in other versions of the sorites paradox.

Delia Graff (2000) provides an account of the sorites paradox that shares many of the virtues of the contextualist account just canvassed, but which promises to evade these kinds of problems. Graff does not explain the force of the sorites by appeal to vague expressions having different semantic contents in different contexts. On Graff's view, in the course of a sorites series each occurrence of a vague term has the same content as the other occurrences. However, in the course of a sorites series, the extensions of the univocal property expressed by the different occurrences of the vague term differ. Let me explain.

7 Thanks to Richard Heck for help with replacing my earlier attempt to formulate this worry with this superior version. 
Consider a sentence such as:

(15) That mountain is tall for a mountain.

Graff adopts the degree theoretic treatment of comparative adjectives developed in Kennedy (1999). According to it, 'tall' denotes a measure function, a function from objects to degrees on a scale (in this case, a scale of height). According to Kennedy, in the syntax of a sentence such as (15) there is an absolute morpheme that combines with the measure function denoted by 'tall' to yield a function from individuals to truth-values. The function takes an individual $x$ to the true just in case the degree of height of that individual (the value of $\operatorname{tall}(x)$ ) is at least as great as the contextually salient degree of height.

To treat the phenomenon of vagueness, Graff proposes adjusting the meaning of Kennedy's absolute morpheme, so that it means significantly greater than, rather than at least as great as (see Graff 2000: 74). She also makes the meaning of 'significantly greater than' relative to persons (75). Abstracting from irrelevant details, on Graff's theory, where $x$ is a person (most naturally taken to be the person who utters the sentence), relative to a context, the proposition expressed by an occurrence of (15) is:

(16) That mountain has significantly greater height for $x$ than the typical height for mountains.

So, in short, Graff's theory exploits Kennedy's account of adjectives, replacing Kennedy's at least as great as relation by her significantly greater than for $x$ relation. ${ }^{8}$

As Graff points out, her proposal involves 'less context dependency' than contextualist accounts of the sorites. Consider the predicate 'significant to Tony Blair'. This predicate is not context-sensitive. It always expresses, as Graff points out, the same interest-relative property. However, since what is in fact significant to Tony Blair changes over time (and across worlds), the univocal property expressed by this predicate changes its extension over time (and across worlds). But this change in extension is due to a change in the facts at different times, not due to features of the context of use of the predicate. Similarly, the predicate 'is a US Citizen' changes its

${ }^{8}$ Graff is admirably explicit about the syntax and semantics of her proposal. However, as she recognizes, the cost of her explicitness is that her proposal faces the worry that it does not generalize to expressions that are not comparative adjectives. For example, no one has ever suggested that in the syntax of nouns such as 'heap' there is a 'greater than' relation, as they have in the case of adjective phrases. But as I have previously emphasized, one's account of the force of the sorites for nouns should be the same as one's account of the force of the sorites for comparative adjectives. There is no plausibility to a thoroughly disjunctive account of the force of the sorites, one for nouns, one for verbs, and one for comparative adjectives. So it is in fact a very serious worry with Graff's proposal that it is not clear how to generalize it to nouns and verbs. 
extension over time, though it always expresses the same property, and so is not context-sensitive. ${ }^{9}$

Here is how Graff's theory accounts for the force of the sorites. Consider a series of people. On the far left is a man who is clearly tall for a typical British man. On the far right is someone clearly not tall for a typical British man. To the immediate right of the man on the far left is a person one millimetre shorter than him, and to his right is a man one millimetre shorter than him, and in general, to the right of each man in the series is someone one millimetre shorter than him, a series stretching until the man on the far right. For each pair of men $x$ and $y$ standing next to one another, we are inclined to accept the conditional:

(17) If $x$ is tall, then so is $y$.

Suppose one person, Hannah, is successively considering all of the conditionals of the form (17). Unlike contextualist solutions, on Graff's account, the property expressed by 'tall' in such a context does not shift when Hannah considers the truth-value of these successive conditionals. In each case, it expresses the interest-relative property of being, for Hannah, significantly taller than the typical British male. But since Hannah considers these conditionals at different times, what counts as significant for Hannah changes at these different times. So, the extension of the interest-relative property of being, for Hannah, significantly taller than the typical British male, changes during the course of her consideration of the different conditionals. So, while the contextualist explanation appeals to shifts in the property expressed by a vague term across contexts to explain a change in extension, Graff's interest-relative account of vagueness appeals to a change in time (circumstance of evaluation) to explain the change in extension, despite no change in the property expressed.

Assuming that Graff can somehow extend her interest relative semantics to noun and verb denotations, she has no problem with a sorites series such as (10), (11), or (12). For her, the property expressed by the ellided Verb Phrase is exactly the same property as was expressed by the initial use of the vague expression. Since it is an interest-relative property, the property has a different extension relative to different times, varying as a function of the relevant person's interests. So, her account of a sorites series involv-

${ }^{9}$ It is instructive to see why Graff needs to relativize the 'significantly greater than' relation to persons. If she did not, then the proposition expressed by (15) would be that the mountain is significantly greater than the typical height of mountains. But then no truth-value for this proposition would be determined given a time and a world. For a time and a world pair is too large to determine what is significant. There are potentially too many conversations at that world and time to fix on a unique set of interests. So one needs to relativize the relation to a particular person or persons. 
ing VP ellipsis is simply just the same as her account for a sorites series not involving VP ellipsis. ${ }^{10}$

Graff's theory also has no problems with the versions of the sorites that involve counterfactual conditionals. Since, on her view, vague terms express properties that concern interests, what will be relevant to fixing the extension of a vague term relative to another possible situation will be the interests we would have in that other possible situation. This yields a satisfactory, uniform account of versions of the sorites such as (14).

Graff's theory correctly reflects the sense that, when one person evaluates a sorites series, the force of the series has nothing to do with a shift in the property expressed by the vague term. However, in order to capture this data, Graff incurs some extremely strong commitments about the propositional content of occurrences of sentences containing vague terms. In particular, since, on her view, vague expressions express interest-relative properties, propositions expressed by occurrences of sentences containing them are what one might call interest-relative propositions. Such propositions are about persons and their interests.

The sense in which the propositions expressed by occurrences of sentences containing vague terms are about persons and their interests is as strong as it can be. As Graff writes:

Significantly greater than is a context-dependent relation, since what is significant to one person may not be significant to another. Any use of 'significant', or of any word whose content involves what is significant, requires an implicit subject with interests - an answer to the question: significant to whom? (2000: 75)

Graff's point here is that 'significant' is what is sometimes called a 'relational word', one that involves implicit anaphora. For those who accept Russellian conceptions of propositions, it should be uncontroversial that the propositions expressed by sentences containing relational expressions that involve implicit anaphora contain, as constituents, the objects or properties in question. ${ }^{11}$ For example, consider the relational word 'enemy', and the proposition expressed by (18), as uttered by Bill:

${ }^{10}$ Instances of the schema ' $x$ is $[\mathrm{F}]$ too' involve an anaphoric presupposition, about something previously mentioned, that it is $\mathrm{F}$, in addition to $x$ being $\mathrm{F}$. The tense of the instance of ' $x$ is F too' filters down to the presupposition, so that the presupposition is that the previously mentioned thing is, at the very same time as $x$ is said to be $\mathrm{F}$, also F. But this does not pose a problem for a Graffian account of a sorites series like (10). The previously mentioned instance is also in the new extension of the property at the later time.

${ }^{11}$ It should also be uncontroversial that the sentences containing expressions involving implicit arguments contain syntactic elements whose value, relative to a context, is the implicit argument. For example, this position can be bound ('Most people have a 
(18) John is an enemy.

Like 'significant', the word 'enemy' involves an implicit argument; an enemy is an enemy of $x$, for some contextually understood $x$. The propositions expressed by sentences containing 'enemy' contain the implicit argument as a constituent. For example, the proposition expressed by this occurrence of (18) is that John is an enemy of Bill. Had Bill not existed, what is expressed by (18) could not be true. Similarly, no one could believe what is expressed by (18) without having a belief about Bill.

According to Graff, in the syntax of sentences containing comparative adjectives there is an expression, Kennedy's absolute morpheme, that is to be understood as the significantly greater than relation. So, the absolute morpheme is a relational expression, like 'enemy'. As a consequence, its implicit argument, relative to a context, is a constituent of the proposition expressed by sentences containing it, relative to that context. So, in particular, the propositions expressed by sentences containing comparative adjectives, for Graff, contain, as constituents, the individual or individuals whose interests are at issue.

But this is problematic. Suppose John utters:

(18) Mount Everest is tall for a mountain.

According to Graff, this occurrence of (18) expresses the proposition that Mount Everest is significantly for John taller than the typical mountain. But what (18) expresses could still be true, even if John had never existed (and hence didn't have interests or purposes). Furthermore, one can easily believe what is expressed by (18) without having a belief about John. This suggests, contra Graff, that there is no expression in the syntax of sentences containing comparative adjectives that is relative to the interests of persons.

Even if the implicit argument of the significantly greater than relation were not a constituent of the proposition expressed by (18), there would still be a serious worry for Graff. For even if there were no people, and hence no significant interests at all, what is expressed by an occurrence of (18) could still be true. But it is difficult to see how the proposition expressed by occurrences of (18) on Graff's account could be true in such a circumstance.

In a similar vein, there are instances of the sorites paradox, such as Wang's Paradox, that involve necessarily existing objects and properties that presumably are essential to them. For example, the number 3 is small, and if $n$ is small, then so is $n+1$. Any account of the force of the sorites paradox must explain these as well. But the proposition expressed by ' 3 is

significant other'). But this additional commitment is somewhat more controversial, involving, as it does, differing views about the nature of syntax (see Partee 1989; Stanley 2000). 
small' is true, even in possible situations in which there are no significant interests.

One response Graff could give to these problems would be to rigidify on the significant interests of the person in question. The aim of this tactic would be to have the above envisaged occurrence of (18) express something like:

(19) Mount Everest is actually for John significantly greater in height than the typical mountain.

If this tactic is successful, then perhaps the modal pressure on Graff's proposal would be alleviated. For what (19) expresses could be true in a world in which John does not exist, since what would be relevant in that world is whether Mount Everest bears the actually-significantly-greater-than-forJohn relation to the height of the typical mountain in that world. ${ }^{12}$

However, this rigidification strategy does not free Graff from the problems. First, it robs Graff of an advantage her theory has over the contextualist. For it leaves her in the same position as the contextualist with respect to versions of the sorites like (14). Secondly, it does not help with the most serious problem facing her view. In order to believe what is expressed by an occurrence of (18) with John as the speaker, one does not need to believe anything so fanciful as (19). One can believe what is expressed by such an occurrence of (18), without having beliefs about either John or what is significant to him, and one can believe what is expressed by such an occurrence of (18) without having any beliefs about the value of the rigidifying operator 'actually'. So Graff's proposal gives an inadequate account of the content of the propositions expressed by sentences containing vague terms. ${ }^{13}$

According to contextualist accounts of the sorites, in evaluating a sorites series, the property expressed by a vague term changes as the context of evaluation changes. But such accounts cannot explain the force of a sorites series where the semantic mechanisms postulated by the contextualists are inoperative. Since a uniform account of the force of the sorites is desired,

12 Thanks to Delia Graff for this suggestion. An independent worry about this strategy, which I will not develop, is that it is not clear how to rigidify. Where does the actually operator occur in the syntax?

${ }^{13}$ Here is another worry for Graff's proposal, due to Timothy Williamson (p.c.). Consider a version of the sorites paradox the major premiss of which is 'For all $x, y$, and $\mathrm{t}$, if $x$ is tall at time $t$, and $y$ is one millimeter shorter than $x$ at $t$, then $y$ is tall at $t^{\prime}$. It is not clear how to extend Graff's proposal to meet this version of the paradox, where the time of evaluation is explicit. However, Graff may respond by arguing that the significantly greater than for $x$ relation has a temporal element that is potentially distinct from the temporal element associated with 'tall' (or the copula), and instances of this universal generalization will involve different times associated with the significantly greater than for $x$ relation. There are problems with this response, but I will not pursue these issues here. 
contextualist solutions are problematic. Graff's interest-relative account can give the desired uniform account of these cases, because her account of the sorites does not involve the property expressed by a vague term changing as one proceeds down the sorites series. But to achieve this result, she must enrich the properties expressed by vague terms with information about persons and their interests, which has highly counter-intuitive consequences in modal and epistemic contexts. I conclude that neither contextualist nor interest-relative accounts of the force of sorites satisfactorily explain its grip. ${ }^{14}$

\author{
University of Michigan, Ann Arbor \\ Ann Arbor, MI 48109-1003, USA \\ jasoncs@umich.edu
}

\title{
References
}

Graff, D. 2000. Shifting sands: an interest-relative theory of vagueness. Philosophical Topics 28: 45-81.

Kamp, H. 1981. The paradox of the heap. In Aspects of Philosophical Logic, ed. U. Monnich. Dordrecht: Reidel.

Kennedy, C. 1999. Projecting the Adjective: The Syntax and Semantics of Gradability and Comparison. New York: Garland Press.

Ludlow, P. 1989. Implicit comparison classes. Linguistics and Philosophy 12: 521-33.

Partee, B. 1989. Binding implicit variables in quantified contexts. In Papers from CLS, ed. C. Wiltshire, B. Music, and R. Graczyk, 25: 342-65. Chicago: Chicago Linguistics Society.

Raffman, D. 1994. Vagueness without paradox. Philosophical Review 103: 41-74.

Raffman, D. 1996. Vagueness and context-sensitivity. Philosophical Studies 81: 175-92.

Robertson, T. 2000. On Soames's solution to the sorites paradox. Analysis 60: 328-34.

Soames, S. 1999. Understanding Truth. New York: Oxford University Press.

Soames, S. 2002. Replies. Philosophy and Phenomenological Research 62: 429-52.

Stanley, J. 2000. Context and logical form. Linguistics and Philosophy 23: 391-434.

Stanley, J. 2002. Nominal restriction. In Logical Form and Language, ed. G. Peters and G. Preyer, 365-88. Oxford: Oxford University Press.

Stanley, J. and Z. Szabo. 2000. On quantifier domain restriction. Mind and Language 17: 219-61.

Tappenden, J. 1993. The liar and sorites paradoxes: toward a unified treatment. Journal of Philosophy 90: 551-77.

${ }^{14}$ My greatest thanks for discussion of the topics in this paper and comments on drafts go to Delia Graff, Richard Heck and Timothy Williamson. I gave this paper at the Universities of Reading and Glasgow, as well as David Charles's reading group at Oxford. Comments by the audiences at all three venues helped. Finally, this paper was written while enjoying a Visiting Fellowship at New College, Oxford - I am grateful to the members of that institution for the temporary use of their wonderful facilities, such as their philosophers. 